\title{
Role of MRI Spectroscopy in diagnosis of brain Glioma: A Cross-Sectional Study
}

\begin{abstract}
Ahmed Okasha ${ }^{a} *$, Ali R. Hamdan ${ }^{b}$
${ }^{\mathrm{a}}$ Department of Diagnostic and Intervention Radiology, Faculty of Medicine, South Valley University, Qena 83523, Egypt.
\end{abstract}

${ }^{\mathrm{b}}$ Department of Neurosurgery, Faculty of Medicine, South Valley University, Qena 83523, Egypt

\begin{abstract}
Background: Glioma a common type of the brain tumor, which originates in the glial cells that surround and support neurons in the brain, including astrocytes, oligodendrocytes and ependymalcells.

Objectives: This study is aiming to investigate the role of MRS in patients in diagnosis of brain glioma.

Patients and methods: The study was designed as a cross-sectional study recruit 30 patients, who were referred to the Radio-diagnosis Department of South Valley University Hospitals for suspected glioma from neurosurgery department during the period from February 2018 to Aug 2021 the study protocol was approved by the local ethics committee of South valley University hospitals (Ref No Code Ms-4-2018, Date of approval 10-02-2018). and it was approved by the local Research Ethics Committee of our hospital.

Results: The study included 30 patients with age ranged from six to 72 years old.

The lesions within the repetitive tumor group had essentially higher $\mathrm{Cho} / \mathrm{Cr}$ ratios than those within the radiation damage group $(\mathrm{p}<0.0001 ; \mathrm{SE}, \quad 0.18)$, and these, also, had vitally higher Cho/Cr proportions than the typical white matter of 6 patients $(\mathrm{p}=0.0003$; SE, 0.09). In differentiating tumor versus non-tumor lesions, the sensitivity and specificity of Cho/NAA ratio greater than 2.0 was $96 \%$ and $70 \%$, respectively. For ratios higher than 2.5 , the sensitivity and specificity to detect tumors was $97 \%$ and $86 \%$, respectively.

Conclusion: MRS examination was greatly beneficial in differentiating neoplastic from non-neoplastic lesions and decrease for stereotactic brain biopsy. However, further studies are required to confirm the current evidence.
\end{abstract}

Key words: MRI Spectroscopy, Brain glioma.

\section{Introduction}

Glioma is a tumor that develops from differentiated glial cells from neuroectoderm and accounts for around $60 \%$ of all primary brain cancers. There are high-grade gliomas (WHO grade III-IV) that have a poor prognosis and are difficult to remove surgically (Xiong et al, 2019). Overall survival (OS) of glioblastoma patients is 14-16 months on average. Histopathology is now the gold standard for detecting glioma recurrences (Gilbert et al, 2013). Radiation treatment and immunotherapy are among the modern approaches for treating gliomas (Reznik et al, 2018).
Variables such as the location of the recurrence, the Karnofski performance score (KPS), and firsttreatment effects influence glioma recurrence (van Linde et al, 2017).

Every surgical operation, in general, has a considerable risk, which may be represented as mortality and morbidity. Craniotomy has a low death rate of less than $3 \%$, but the total incidence of complications is estimated to be between $6 \%$ and $21 \%$, with a potential of up to $30 \%$ depending on the location and grade of the tumor, amount of resection, and the patient's condition of health (Vives and Piepmeier,1999).

Copyright: (c) Okasha and Hamdan (2022) Immediate open access to its content on the principle that making research freely available to the public supports a greater global exchange of knowledge. Users have the right to Read, download, copy, distribute, print or share link to the full texts under a Creative Commons BY-NC-SA 4.0 International License. 
When it comes to the overall morbidity of stereotactic brain biopsies, they are nevertheless substantial, even if they are a minimally invasive technique with low mortality (less than 1\%)(Hall ,1998). This non-invasive diagnostic technique is becoming more effective due to recent advances in advanced magnetic resonance imaging technologies. Glioma identification and grading by standard MR imaging, on the other hand, might be controversial since glioma grading ranges from 55 to $83 \%$ in sensitivity (Law et al, 2003). In the tumor tissue, variations in metabolite concentration indicate changes in tumor cell metabolism associated with malignant transformation. In light of glial tumor metabolite features that vary by grade, there is an increasing interest in MRspectroscopy (MRS), which can enhance commonly utilized imaging (Horská and Barker, 2010).

In this study, we aimed to assess role of MRS in diagnosis of brain glioma.

\section{Patients and methods}

The study population included thirty patients, who were referred to the Radio-diagnosis department in south valley University hospitals for suspected glioma from neurosurgery department during the period from February 2018 to Aug 2021 the study protocol was approved by the local ethics committee of South valley University hospitals (Ref No Code Ms-4-2018, Date of approval 10 02-2018). and it was approved by the local Research Ethics Committee of our hospital. All the participants were told about the details and gave a written consent.

Inclusion criteria: patients have space occupying lesion on Brain CT and MRI with criteria suggested glioma.

Exclusion criteria: patients with metastatic lesions and patients or parents/caregiver who refused to sign the informed consent. Besides, we excluded severely dyspneic patients with motion artefacts on MRI

Data collection and MRS technique: We collected the demographic characteristics, final diagnosis, and findings of MRI spectroscopy. The final diagnosis was obtained by a variety of testing panels, included cerebrospinal fluid (CSF) analysis, radio-isotope study, CT, MRI, and/or lumbar puncture, cytology, and histopathology.

The conventional MRI and MRI spectroscopy was performed using 1.5-T unit (Achieva, Philips)in the supine position. In case of conventional MRI, the $0.1-0.2 \mathrm{mmol} / \mathrm{kg}$ IV Gd-DTPA was used as a contrast media. All patients underwent routine spin echo (SE) sequences, followed SE-T2-weighted images for single voxel (SVS) using Point Resolved Spectroscopy (PRESS) sequence to select the volume of interest (VOI). T1WI, T2WI and FLAR sequences were done. Multi-voxel spectroscopy was performed for all patients. The used protocols for conventional MRI and MRI spectroscopy are present in Table 1 . In Table 2, we showed the concentration of each chemical metabolite within the brain lesion on MRI spectroscopic analysis.

\section{Table 1. The Used MRS and MRI Protocols}

\begin{tabular}{|c|c|}
\hline Conventional MRI & MRI spectroscopy \\
\hline 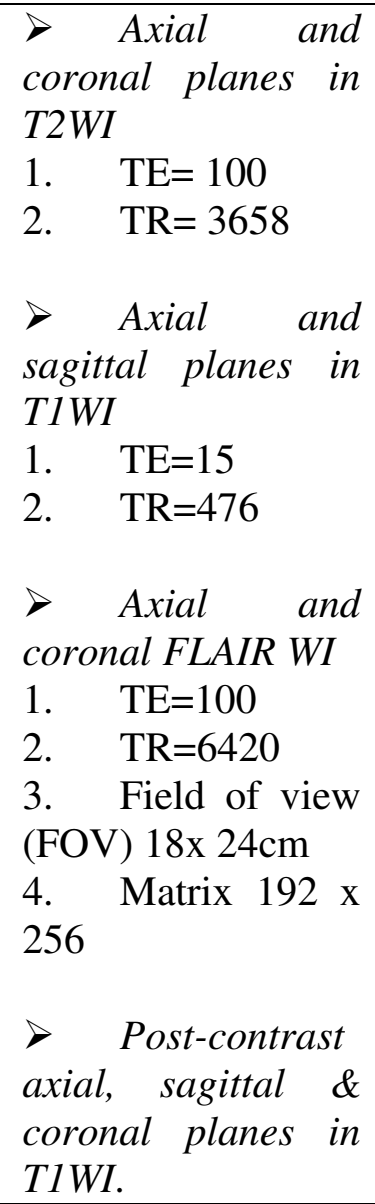 & $\begin{array}{l}\text { Axial, sagittal \& } \\
\text { coronal planes in } T 1+C \text { or } \\
\text { T2WI MRS in } \\
\text { 1. long } \mathrm{TE}=288 \text { (multi- } \\
\text { voxel) } \\
\text { 2. Short } \mathrm{TE}=31 \text { (single } \\
\text { voxel) } \\
\text { Intermediate } \mathrm{TE}= \\
\text { 144 (single voxel) } \\
\text { 4. With } \mathrm{TR}=2000\end{array}$ \\
\hline
\end{tabular}


Table 2. The concentration of each metabolite on MRI Spectroscopic Analysis

\begin{tabular}{|c|c|c|c|c|c|c|}
\hline Diagnosis & Cho & $\boldsymbol{C r}$ & $\boldsymbol{G l x}$ & NAA & Lipid & Lactate \\
\hline $\begin{array}{c}\text { Glioblast } \\
\text { oma } \\
\text { GBS }\end{array}$ & - -to++ & -- & -- & -- & +++to++++ & +++to++++ \\
\hline $\begin{array}{c}\text { Glioma } \\
\text { (other) }\end{array}$ & +++ to & - to-- & -- & - to--- & Absent to + & Absent to ++ \\
\hline $\begin{array}{c}\text { Radiation } \\
\text { necrosis }\end{array}$ & $\begin{array}{c}\text { Absent } \\
\text { to }\end{array}$ & $\begin{array}{c}\text { Absent } \\
\text { to }\end{array}$ & $\begin{array}{c}\text { Absent } \\
\text { to }\end{array}$ & $\begin{array}{c}\text { Absent } \\
\text { to }\end{array}$ & $\begin{array}{c}\text { Absent to } \\
++++\end{array}$ & Absent to ++ \\
& -- & -- & -- & -- & & \\
\hline
\end{tabular}

Semi quantitative 9-point scale runs from ---- to normal to ++++

\section{- indicates decreased from normal,}

+ indicates increased from normal

\section{Lipid and lactate are normally absent}

\section{Statistical Analysis:}

-Continuous data with normal and abnormal distribution were expressed as mean $( \pm$ SD) and median (interquartile range $[\mathrm{IQR}]$ ) values, respectively. Categoric data were expressed as a count and percentage

\section{Results:}

The study included 30 patients. 17 female (56.7\%) and 13 male (43.3).with age ranged from six to 72 years old. The distribution of the final diagnosis was as follows: oligodendroglioma $(n=3)$, low grade glioma $(\mathrm{n}=10)$, glioblastoma multiformis $(n=5)$.Post-irradiation tumoral residue $(n=5)$. Postirradiation necrosis $(n=6)$, brain stem glioma $(n=1)$. Thus, a total of 24 has neoplastic lesion.

The histopathological confirmation was obtained in low grade glioma in 10 patients, glioblastoma multiformis in four patients, oligodendroglioma in three patients, post-radiation residual tumor in 5 patient, post-radiation necrosis in six patients, the diagnosis was confirmed by the response to the treatment, follow-up CT and/or MRI, radio-isotope study, or lumbar puncture and cytology. Only one pathologically diagnosed patient to be glioblastoma multiformis that did not match our suggested diagnosis by MRS (which was a non-neoplastic process) and this was attributed to technical reasons (voxel contamination by surrounding normal parenchyma due to small lesion).

The following statistical assessment was gotten using the generalized estimating equation technique. The lesions within the repetitive tumor group had essentially higher $\mathrm{Cho} / \mathrm{Cr}$ ratios than those within the radiation injury group $(\mathrm{p}<0.0001$; SE, 0.18), and these, also, had vitally increased $\mathrm{Cho} / \mathrm{Cr}$ ratios than the typical white matter of 6 patients ( $p=0.0003$; SE, 0.09). The lesions in the in the radiation injury group $(\mathrm{p}<0.0001$; SE,0.32) had essentially lower Cho/NAA ratios than those in the tumor group and also the radiation group had significantly higher Cho/NAA ratios than the normal-appearing white matter of 26 patient $(\mathrm{p}<$ 0.0001; SE, 0.08). Lesions in the in the radiation injury group had significantly higher $\mathrm{NAA} / \mathrm{Cr}$ ratios ( $\mathrm{p}<0.0001$; SE, 0.09) than those in the tumor group. The average values of the $\mathrm{Cho} / \mathrm{Cr}$, Cho/NAA, and NAA/Cr ratios in particular lesions and typical white matter are summarized in Table 3.

In differentiating tumor versus non-tumor lesions, the sensitivity and specificity of Cho/NAA ratio greater than 2.0 was $96 \%$ and $70 \%$, respectively. For ratios higher than 2.5, the sensitivity and specificity to detect tumors was $97 \%$ and $86 \%$, respectively. 
Table3: Ratios between Choline, Creatine, and NAA in Recurrent Tumor, radiation injury and normal appearing white matter.

\begin{tabular}{|l|c|c|c|}
\hline Ratio & Tumor Tissue & Radiation Injury & $\begin{array}{c}\text { Normal } \\
\text { Appearing } \\
\text { White Matter }\end{array}$ \\
\hline $\boldsymbol{C H O / C R}$ & $2.52(1.66-4.26)$ & $1.57(0.72-1.76)$ & $1.14(0.86-1.59)$ \\
\hline $\boldsymbol{C H O / N A A}$ & $3.48(1.70-6.47)$ & $1.31(0.83-1.78)$ & $0.79(0.56-1.2)$ \\
\hline NAA/CR & $0.79(0.47-1.15)$ & $1.22(0.94-1.69)$ & $1.39(0.64-2.0)$ \\
\hline
\end{tabular}

Note-Data are mean ratios (ranges in parentheses). Cho = choline, $\mathrm{Cr}=$ creatine, NAA = N-acetyl aspartate

\section{Cases Presentation}

\section{Case 1.}

53year-old Male patient presented by chronic headache. Imagining modalities Fig.1(A) Axial T2WI right fronto-temporal ill-defined intra axial mass lesion of high signal with areas of break down and surrounded by moderate vasogenic edema. (B) $\mathrm{T} 1 \mathrm{~W}$ post. Contrast showing no obvious enhancement.(C)Intermediate echo spectroscopy curve shows Elevated Choline, lipid/lactate peak as well as MI were noted on short TE with reversal of lactate peak. Cho/Cr ratio 2.5, Cho/NAA ratio 6.2.

Collectively, the findings are in favor of grade II Glioma in view of presence of high MI peak. Histopathological examination confirmed the diagnosis: Astrocytoma (grade II).Fig. 1.

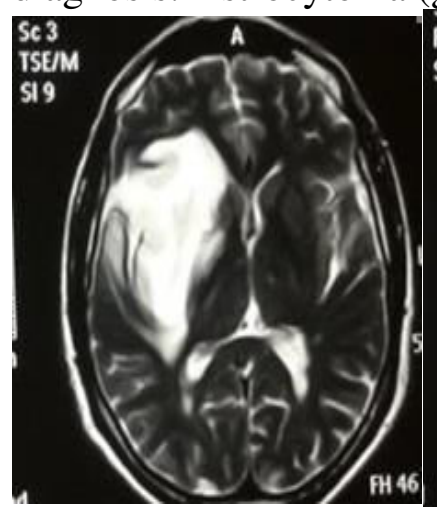

A

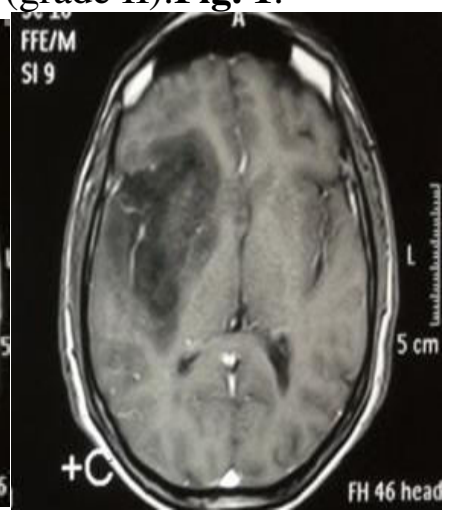

B

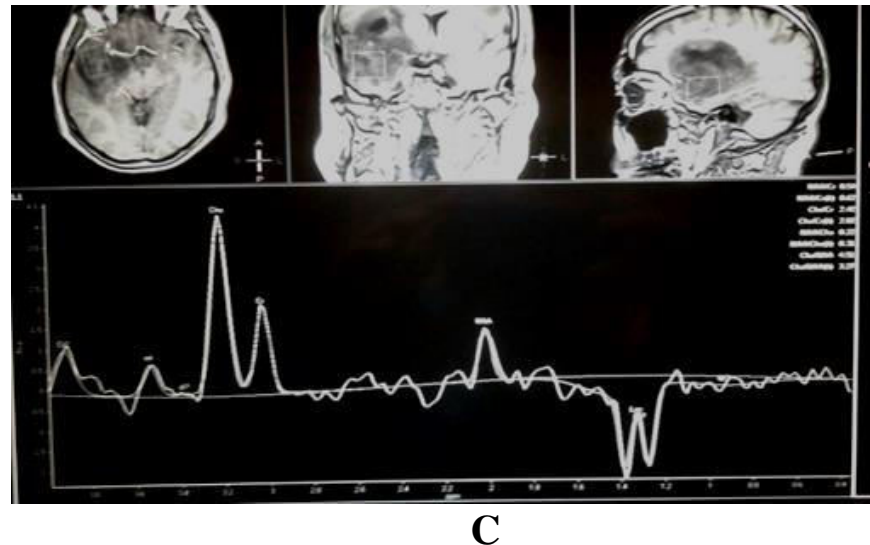

Fig.1(A).Axial T2 right fronto-temporal ill-defined intra axial mass lesion of high signal with areas of break down and surrounded by moderate vasogenic edema.(B) $\mathrm{T} 1$ post.contrast showing no obvious enhancement.(C) Intermediate echo spectroscopy curve shows Elevated Choline, lipid/lactate peak as well as MI were noted on short TE with reversal of lactate peak. Cho/Cr ratio 2.5, Cho/NAA ratio 6.2.

\section{Case 2.}

7-year-old female patient presented by chronic convulsions. Fig.2(A).Axial T2 show left parietal ill-defined intra axial mass lesion of high signal. (B)AxialT1 post-contrast showing faint heterogeneous enhancement. Intermediate echo. (C) spectroscopy curve shows increased level of MI, Cho with reduced NAA, no lipid or lactate.

Collectively, the findings are in favor of low-grade glial neoplasm, DNET was excluded in view of decreased NAA/ Cho ratio (which is normal in 
DNET). Histopathological examination confirmed the diagnosis: Low grade Astrocytoma.Fig. 2.

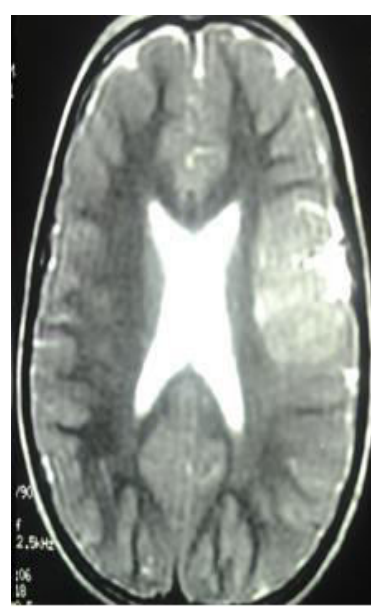

A

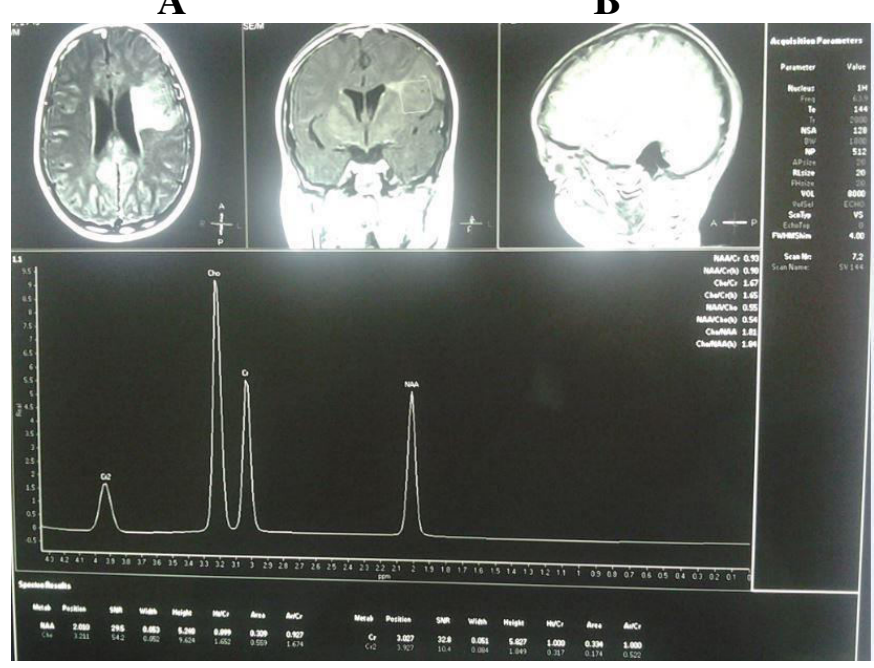

C

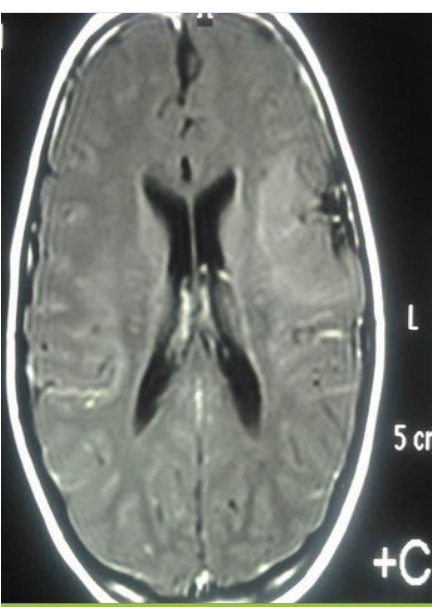

B tal ill-defined

Fig.2 (A) Axial T2 show left parietal ill-defined intra axial mass lesion of high signal. (B)Faint heterogeneous enhancement in post contrast T1WI. (C)Intermediate echo spectroscopy curve shows increased level of MI, Cho with reduced NAA, no lipid or lactate.

\section{Case 3.}

20-year-old female patient presented by headache, vomiting, and blurring of vision, fundus examination revealed papilledema. Fig.3 (A)Axial T2 show a hyperintense ill-defined mass lesion is seen epicentered at left thalamus \& encroaching upon the 3 rd ventricle \& quadrigeminal cistern, compresses left mid brain hemi portion. (B)AxialT1 post-contrast showing marginal heterogeneous enhancement.(C)Intermediate echo spectroscopy curve shows Elevation of Cho with reduction of $\mathrm{Cr}$ and NAA, Lipid/lactate peak was reversed.

Collectively, the findings are in favor of high grade glial neoplastic mass (Thalamic glioma).
Histopathological examination confirmed the diagnosis: Thalamic glioma. Fig. 3.

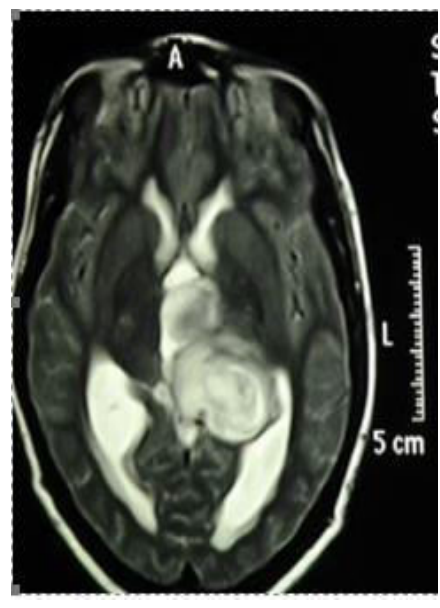

A

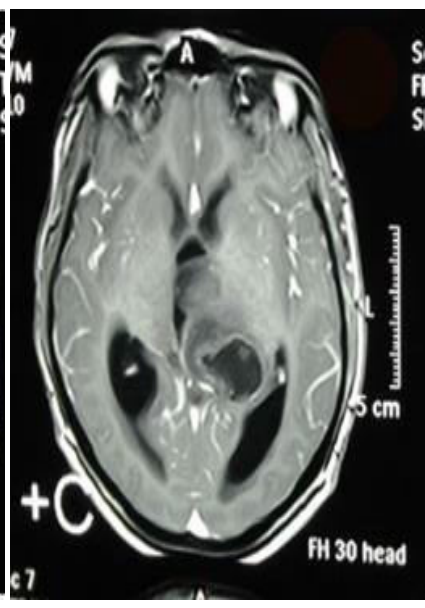

B

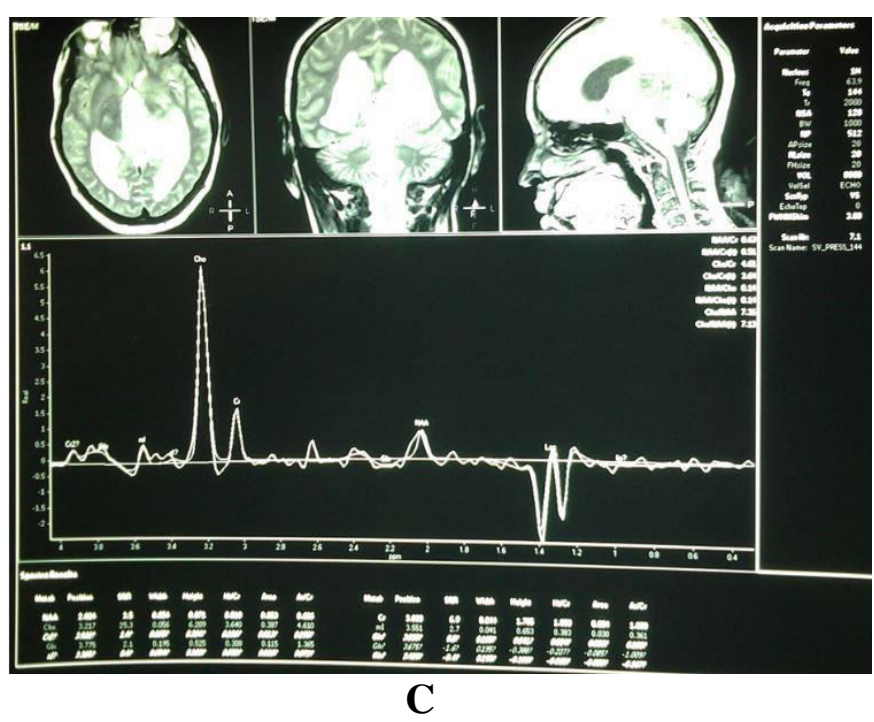

Fig.3 (A). Axial T2 show a hyperintense ill-defined mass lesion is seen epicentered at left thalamus \& encroaching upon the 3rd ventricle \& quadrigeminal cistern, compresses left mid brain hemi portion. (B). AxialT1 post Gd showing marginal heterogenous enhancement.(C)Intermediate echo spectroscopy curve shows Elevation of Cho with reduction of $\mathrm{Cr}$ and NAA, Lipid/lactate peak was reversed.

\section{Discussion:}

MR spectroscopy is a method for studying a few metabolites in the brain or neoplasm without removing them from the patient. Proton MRS may be able to distinguish between lesions with comparable cMRI features (Hellström et al, 2018). When combined with cMRI, its accuracy ranges from 95 to 100 percent in identifying neoplastic and non-neoplastic lesions, enhancing diagnostic specificity (Cecil ,2013). Patients with intracranial tumors require an accurate diagnosis in order to 
receive the best possible treatment. Most tumors are surgically removed when they are accessible, but there is a delicate balance between removing as much tumor tissue as feasible and protecting essential brain processes (Howe and Opstad KS, 2003).

In our study, the MRS examination was greatly beneficial in in diagnosis of brain glioma, which was our main aim, thus altering the subsequent management and saving some patients the need for biopsy taking. Furthermore, our findings showed that Choline, NAA, Cr, occasionally MI, and lipid/lactate peaks are the most important metabolites in the long term.

As Smith et al., 2003, noted, lesions of the brain stem provide a unique clinical challenge. Most patients are treated based on clinical and imaging findings without biopsy due to the dangers of biopsy in this area. Because of the necessity for small voxels and the magnetic susceptibility changes at the skull base, MRS in this area is especially difficult. Using MRS, the scientists showed that neoplastic and non-neoplastic processes might be differentiated in many cases when all metabolites are taken into account. We agree with Smith and his colleagues since we confronted this difficulty in 1 patient with brain stem lesions, and the final diagnosis was based on the MRS findings and follow-up by cMRI, which verified the diagnosis, and the therapy was initiated accordingly.

As far as tumors are concerned, Cho is the most critical metabolic marker. As tumor cells proliferate, Cho levels rise, resulting in increased cell membrane turnover and cell density (9). Grade II and III gliomas are more likely to have greater Cho levels than glioblastoma multiforme (GBM), which may have lower Cho levels due to necrosis (Tsougos I et al, 2012). While necrosis should be avoided, it is vital to incorporate as much solid lesion as possible in the voxel. The $\mathrm{Cho} / \mathrm{Cr}$ and Cho/NAA ratios should be elevated if there is any evidence of neoplasms (Weybright $P$ et al.,2008). Our study noted that the pathologically proven cases of GBM showed high levels of Cho/NAA ratios, where the least level was 6.4. As for other glial tumors, variable values of increased Cho/NAA ratio were noted. Grade II glial neoplasms, the Cho/NAA ratio was as low as 1.2, while only one of them reached as high as 20 . To illustrate, Cho/NAA was as high as 17.6 in the case of mixed-grade astrocytoma (grade II \& III), compared to just 1.3 in oligodendroglioma. According to Adamson et al. (1998), a Cho/NAA ratio greater than 1 indicates the presence of malignancy. The Cho/NAA ratio greater than 2.0 had a $96 \%$ sensitivity and $70 \%$ specificity in distinguishing tumor from non-tumor lesions; in the study of Mcknight and Von Dem Bussche ( 2010). Over 2.5, tumor detection sensitivity drops to $90 \%$, while specificity increases to $68 \%$. In our study, we could not depend mainly on the fact that Cho/NAA ratio greater than 1.0 or 2.0 was considered to be positive for malignancy as some lesions were borderline, e.g., grade II glioma and oligodendroglioma where the values were 1.2 and 1.3 respectively. Therefore, the diagnosis was suggested based on the $\mathrm{Cho} / \mathrm{Cr}$ ratio level, which was calculated on both the side of the lesion and the contralateral normal side.

Brandão and Castillo (2016) noted that tumors generally had a lower NAA and $\mathrm{NAA} / \mathrm{Cr}$ ratio, indicating reduced vitality and number of neurons.

On the other hand, we could not discover that the NAA/Cr proportion shifts significantly between tumors and the contralateral typical side. We resulted that the ratio reduced in many tumors but increased in one case of oligodendroglioma and remained fixed in one case of low-grade glioma.

MRS has some limitations, including movements and irritability of the patient resulted in false results due to voxel position changes, which made it necessary to repeat the examination, resulting in additional time consumption. Reassuring or even sedated the patient can help alleviate this problem. Anatomic coverage is limited with the single-voxel method. Voxel positioning is therefore crucial, but MVS might help to minimize this restriction. A single voxel could not be used to assess small lesions $(<1 \mathrm{~cm})$ since the voxel would be contaminated by the surrounding normal parenchyma, resulting in a less accurate diagnosis, which also can be resolved with MVS. In addition, we should avoid blood, blood products, air, cerebrospinal fluid (CSF), fat, necrotic regions, metal calcification, and bone to get high-quality spectra.

\section{Conclusion}

MRS examination was greatly beneficial in differentiating neoplastic from non-neoplastic lesions and decrease for stereotactic brain biopsy. 
However, further studies are required to confirm the current evidence.

\section{References}

Adamson AJ, Rand SD, Prost RW, Kim TA, Schultz C, Haughton VM (1998). Focal brain lesions: Effect of single-voxel proton MR spectroscopic findings on treatment decisions. Radiology, 209(1):73-8. doi: 10.1148/radiology.209.1. 9769815.

Brandão LA, Castillo M. (2016). Adult Brain Tumors: Clinical Applications of Magnetic Resonance Spectroscopy. Magnetic Resonance Imaging Clinics of North America,24(4):781-809.

Cecil KM (2013). Proton magnetic resonance spectroscopy: technique for the neuroradiologist. Neuroimaging Clin N Am, 23(3):381-92.

Gilbert MR, Wang M, Aldape KD, Stupp R, Hegi ME, Jaeckle KA, et al (2013) Dose-dense temozolomide for newly diagnosed glioblastoma: A randomized phase III clinical trial. J Clin Oncol,;31(32):4085-91.

Hall WA (1998) The safety and efficacy of stereotactic biopsy for intracranial lesions. Cancer, 82( 9) :17491755.

Hellström J, Romanos Zapata R, Libard S, Wikström J, Ortiz-Nieto F, Alafuzoff I, et al (2018) The value of magnetic resonance spectroscopy as a supplement to MRI of the brain in a clinical setting. PLoS One, 13(11):e0207336.

Horská A, Barker PB (2010) Imaging of brain tumors: MR spectroscopy and metabolic imaging. Neuroimaging Clinics of North America, 20(3):293-31. ( doi: 10.1016/j.nic.2010.04.003)

Howe FA, Opstad KS (2003) $1 \mathrm{H} \quad \mathrm{MR}$ spectroscopy of brain tumours and masses. NMR Biomed 16(3):123-31.

Law M, Yang S, Wang H, Babb JS, Johnson G, Cha S, et al (2003) Glioma Grading: Sensitivity, Specificity, and Predictive Values of Perfusion MR Imaging and Proton MR Spectroscopic Imaging Compared with Conventional MR Imaging. Am J Neuroradiol, 24(10):1989-98.

Mcknight TR, Von Dem Bussche MH VD (2010) Correlation of 3D ERM imaging tumor index with histology in patients with newly diagnosed gliomas. AJNR, 22:959-968.

Reznik E, Smith AW, Taube S, Mann J, Yondorf MZ, Parashar B, et al (2018) Radiation and Immunotherapy in High-grade Gliomas: Where Do We Stand? Am J Clin Oncol, 41(2):197-212.
Smith JK, Castillo M, Kwock L (2003). MR spectroscopy of brain tumors. MagnReson Imaging Clin N Am, 11(3):415-29.

Tsougos I, Svolos P, Kousi E, Fountas K, Theodorou K, Fezoulidis I, et al (2012). Differentiation of glioblastoma multiforme from metastatic brain tumor using proton magnetic resonance spectroscopy, diffusion and perfusion metrics at 3 T. Cancer Imaging, 12(3):423-36.

Van Linde ME, Brahm CG, de Witt Hamer PC, Reijneveld JC, Bruynzeel AME, Vandertop WP, et al (2017). Treatment outcome of patients with recurrent glioblastoma multiforme: a retrospective multicenter analysis. J Neurooncol, 135(1):183-92. Vives KP, Piepmeier JM (1999). Complications and expected outcome of glioma surgery. Journal of Neuro-Oncology, 42, 289-302.

Weybright P, Sundgren PC, Maly P, Hassan DG, Nan B, Rohrer S, et al (2005) Differentiation Between Brain Tumor Recurrence and Radiation Injury Using MR Spectroscopy. Am J Roentgenol, 185(6):1471-6.

Xiong L, Wang F, Xie XQ (2019) Advanced treatment in high-grade gliomas. J BUON, 24(2):424-30. 